\title{
ON TORSION OF PRISMS WITH LONGITUDINAL HOLES*
}

\author{
BY \\ CHIH-BING LING \\ Aeronautical Research Laboratory, Taiwan, China
}

\begin{abstract}
This paper presents a method of solution, called the method of images, for the torsion of prisms having one or more longitudinal holes. The method is applicable to prisms of the following four, and only four, sections: a rectangle, an equilateral triangle, an isosceles triangle and a $30^{\circ}-60^{\circ}-90^{\circ}$ triangle. These four sections form a group by themselves.

The solution is obtained by adding to the known solution of a corresponding solid prism without holes a system of harmonic functions which vanish on the entire external boundary of the given section, and besides possess a singularity at the centre of each hole. Such a system of functions may be constructed from Weierstrass' Sigma function and its allied functions.

The solution is illustrated by applying it in detail to a rectangular prism having a central longitudinal hole. Numerical results are shown for the special case of a square prism.
\end{abstract}

Introduction. The torsion of a circular cylinder having longitudinal circular holes, with or without a central hole, has been investigated by Kondo ${ }^{1}$ and by the present writer. ${ }^{2}$ In the present paper, the investigation will be extended to a prism, which is also pierced by such longitudinal holes.

Both problems in fact belong to the same general class of torsion problems dealing with cylinders of multi-connected sections. Analytic solutions of such problems are generally difficult except in some simple cases, and indeed very few solutions have ever been found. It appears, however, that certain prisms of this nature can be solved by adapting to them the method of images. There are altogether four such prisms, the cross sections of which are as follows:

(1) a rectangle, including square as a special case,

(2) an equilateral triangle,

(3) an isosceles right triangle,

(4) a $30^{\circ}-60^{\circ}-90^{\circ}$ triangle.

It is not difficult to show that by reflection about the edges each of the above four sections forms a doubly infinite set of images. Furthermore, it can be shown conclusively by theory of groups ${ }^{3}$ that these four sections are the only ones which form such images.

The images formed in each case are shown in Figs. 1-4 respectively. In each figure the shaded area represents the fundamental region, or the given section of the prism:

*Received August 9, 1950.

${ }^{1} \mathrm{M}$. Kondo, The stresses in twisted circular cylinder having circular holes, Phil. Mag. (7) 22, 1089-1108 (1936).

${ }^{2}$ C. B. Ling, Torsion of a circular tube with longitudinal circular holes, Q. Appl. Math., 5, 168-181 (1947).

${ }^{3}$ W. Burnside, Theory of groups, 2nd ed., Cambridge University Press, 1911, 410-418. 
The regions marked by positive signs represent the images which are formed by an even number of reflections, while the regions marked by negative signs represent those which are formed by an odd number of reflections. Note that any two adjacent regions must have alternate positive and negative signs. The set of points in each figure represents the images due to a given point in the fundamental region.

\begin{tabular}{|c|c|c|c|c|c|}
\hline+ & + & - &.$^{+}$ & - &.+ \\
\hline+ & - & + & - & + & - \\
\hline- & + & - & + & - & + \\
\hline+ & - & + & - & + & - \\
\hline- & + & - & + & - & + \\
\hline+ & - & + & - & + & - \\
\hline
\end{tabular}

FIG. 1. Rectangle.

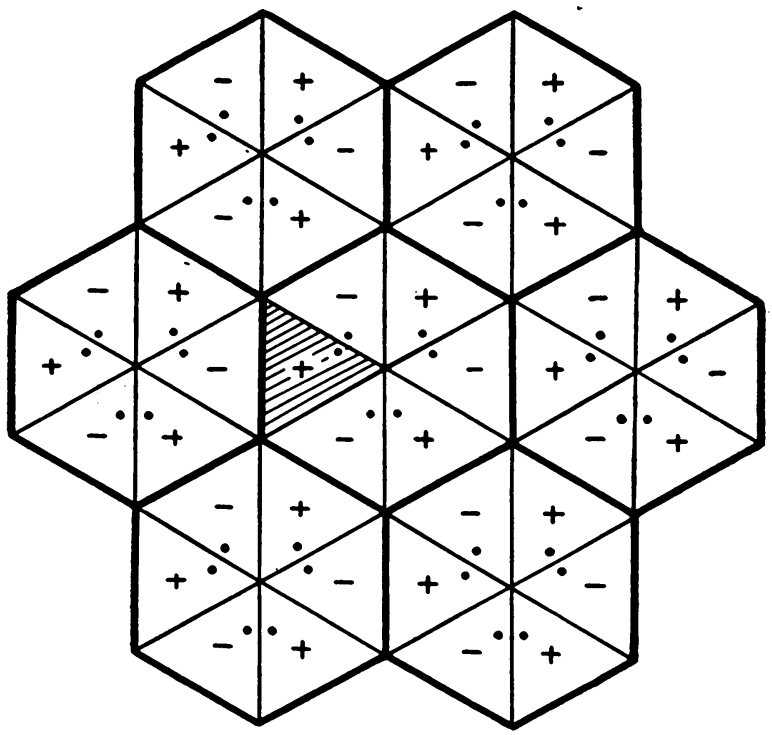

FIG. 2. Equilateral triangle.

It may be noted that in Fig. 1 the rectangles may be regarded as grouping themselves into identical rectangles each composed of four adjacent rectangles. Similarly, in Figs. 2, 3 and 4 the triangles may be regarded as grouping themselves into identical regular hexagons each composed of six adjacent triangles, into identical squares each composed of eight adjacent triangles, and into identical regular hexagons each composed of twelve adjacent triangles respectively. Such identical regions, which are indi- 
cated by heavy lines, form a doubly infinite set in each case. Obviously, such groupings are not unique, but they are immaterial in the present treatment.

Whereas the present solution is restricted to the four sections as mentioned above,

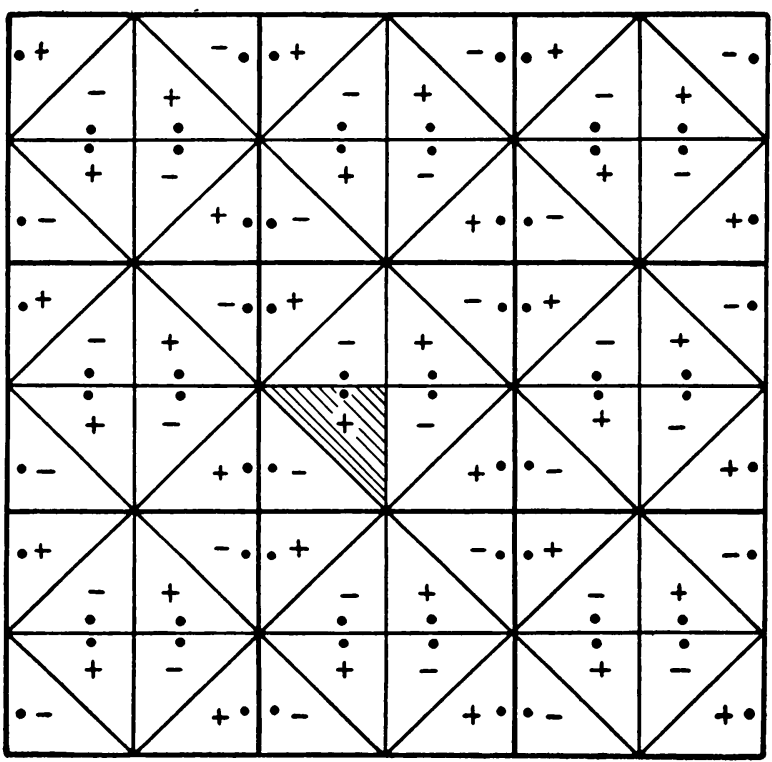

FIG. 3. Isosceles right triangle.

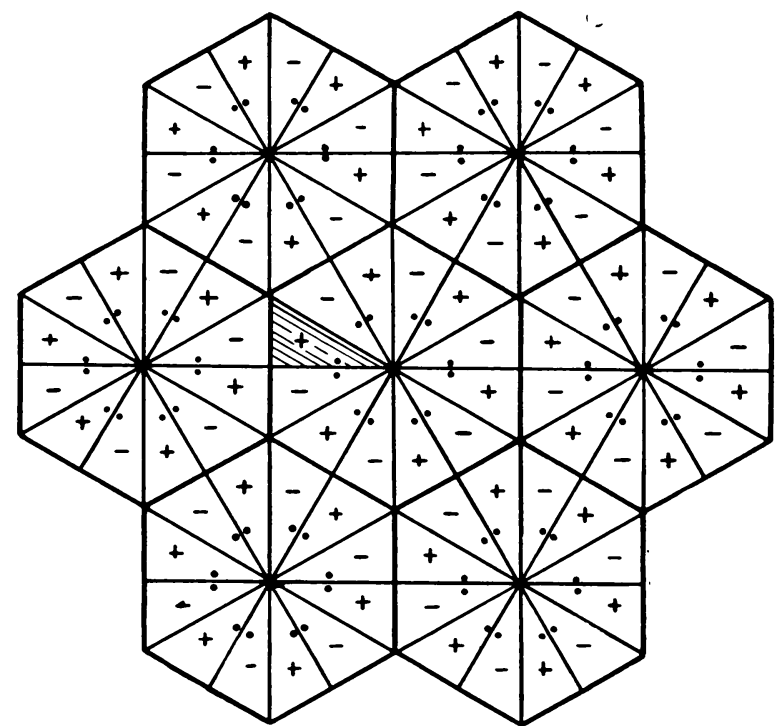

FIG. $4.30^{\circ}-60^{\circ}-90^{\circ}$ triangle.

there is however no further restriction theoretically as to the manner of distribution of the holes within the section. Naturally, the simplest is the case of a single circular hole, especially when the hole is symmetrically located within the section. The next to the 
simplest is a group of similar circular holes. The latter implies that all the holes are of equal radii and symmetrically located within the section so that the properties of all the holes are alike, and consequently if the boundary conditions on any one of the holes are satisfied, the boundary conditions on all the other holes will be automatically satisfied. For convenience, the former will be referred to as the one-hole case and the latter as the invariant case. Various invariant cases are shown in Fig. 5. For the $30^{\circ}-60^{\circ}-90^{\circ}$

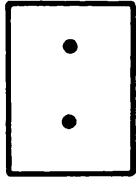

(i)

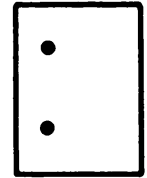

(ii)

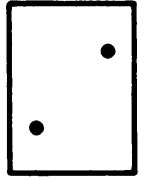

(iii)

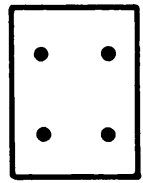

(iv)

(a) Rectangle

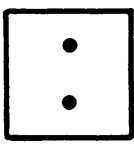

(i)

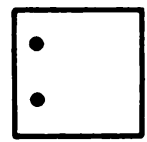

(ii)

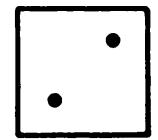

(iii)

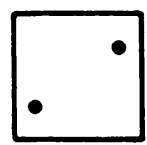

(iv)

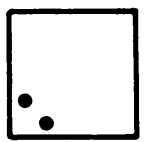

(v)

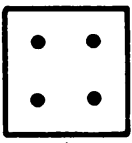

(vi)

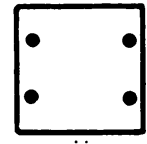

(vii)

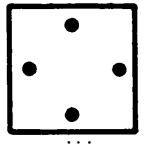

(viii)

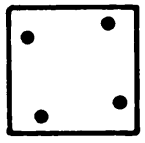

(ix)

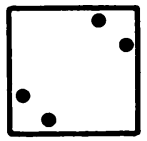

(x)

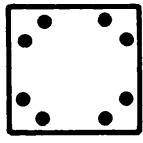

(xi)

(b) Square

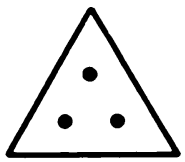

(i)

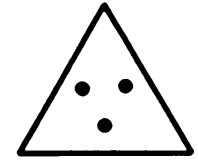

(ii)

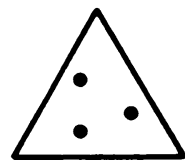

(iii)

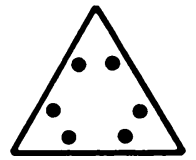

(iv)

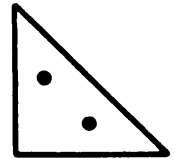

(d) Isosceles right triangle

(c) Equilateral triangle

Fig. 5. Invariant cases (of more than one hole).

triangle, it appears that no such invariant distribution (or more than one hole) is possible.

Method of solution. In general the solution of the torsion problem requires a harmonic function, say $\psi$, whose conjugate function is single-valued, such that the function $\Psi$ defined by the relation

$$
\Psi=\psi-\frac{1}{2}\left(x^{2}+y^{2}\right)
$$

becomes a constant on each boundary of the given section; $x$ and $y$ being the rectangular coordinates in the plane of the section. As no generality is lost, the particular constant on the external boundary will henceforth be taken as zero.

Suppose that the function $\psi$ is composed of two parts as follows:

$$
\psi=\psi_{0}+\psi_{1}
$$


where $\psi_{0}$ represents the solution of a corresponding solid section without holes. This implies that $\psi_{0}$ is a harmonic function which possesses a single-valued conjugate function and is equal to $\frac{1}{2}\left(x^{2}+y^{2}\right)$ on the external boundary of the section. Consequently, the function $\psi_{1}$ must possess the following properties:

(1) it is also a harmonic function,

(2) its conjugate function is also single-valued,

(3) it vanishes on the entire external boundary.

Now, the functions $\psi_{0}$ for a solid prism of the four particular sections mentioned above are well known. ${ }^{4}$ In particular, a solution has been given in a unified manner by Hay. ${ }^{5}$ His method of solution is also described as a method of images, but is essentially based on a different consideration.

The present problem is thus reduced to find a function $\psi_{1}$ which possesses these properties, or more precisely, to find a complete system of such functions for $\psi_{1}$ so that the group of parametric coefficients attached to them can be further adjusted to satisfy the remaining boundary conditions at the internal boundaries of the given section. In addition, since the function $\psi_{0}$ for a solid section possesses no singularity inside the boundary, therefore the function $\psi_{1}$ must be of a different system such that it possesses

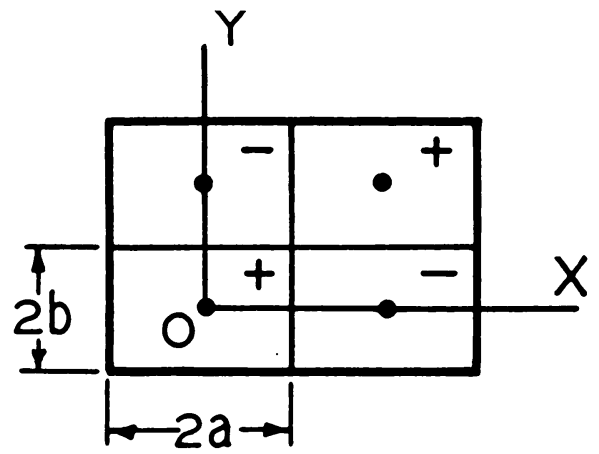

FIG. 6.

singularities inside the external boundary. Such singularities will eventually be excluded from the material of the section by the internal boundaries or holes. Thus, if the holes are circular, they are preferably placed at the centres of the holes. Naturally, the system of functions will be considerably simplified whenever the single hole or the group of holes are symmetrically located within the given section.

It appears that the system of functions for the four sections mentioned above may be constructed from Weierstrass' Sigma function and its allied functions. However, as the sections are so different in nature, it seems that no general expression can be put forward for all the four sections as a whole. In the following, the case of a rectangular prism having a central longitudinal circular hole will be chosen as an illustration. The system

4St. Venant, Mém. des Savants étrangers 14, 233-560 (1856), for rectangle and equilateral triangle.

B. G. Galerkin, Bull. de l'Acad. des Sci. de Russie 13, 111-118 (1919) for isosceles right triangle.

G. Kolossoff, Comptes Rendus 178, 2057-2060 (1924), for isosceles right triangle.

B. R. Seth, Q. J. of Math. 5, 161-171 (1934), for equilateral triangle, isosceles right triangle and $30^{\circ}-60^{\circ}-90^{\circ}$ triangle.

${ }^{5}$ G. E. Hay, Proc. of London Math. Soc. 45, 382-397 (1939). 
of functions will first be constructed. Subsequently, formulas for shear stresses and torsional modulus will be derived. Methods will also be given for evaluating the coefficients or functions involved in the solution. Finally, numerical results will be shown for the special case of a square prism.

Rectangular prism having a central longitudinal hole. A class of doubly periodic functions. Consider in the $z$-plane an identical rectangle composed of four adjacent rectangles, including the fundamental rectangle, as shown previously in Fig. 1. Let the dimensions of each rectangle be equal to $2 a \times 2 b$ and the origin of the coordinates be at the centre of the fundamental rectangle as shown in Fig. 6 . Now consider the doubly infinite set of image points due to a point at the origin. Since the identical rectangle is of double periods $4 a$ and $4 i b$, the affixes of the set of image points in the positive regions, including the given point in the fundamental rectangle, are given by

and

$$
\left.\begin{array}{l}
P_{m n}=4 m a+4 n i b \\
Q_{m n}=2(2 m+1) a+2(2 n+1) i b=P_{m n}+2 a+2 i b,
\end{array}\right\}
$$

where $m$ and $n$ are extended to all positive and negative integers including zero. Again, the affixes of the set of image points in the negative regions are given by

and

$$
\left.\begin{array}{l}
P_{m n}^{*}=2(2 m+1) a+4 n i b=P_{m n}+2 a \\
Q_{m n}^{*}=4 m a+2(2 n+1) i b=P_{m n}+2 i b,
\end{array}\right\}
$$

where $m$ and $n$ are extended to the same range of values as before.

A function with a logarithmic singularity at each such point will be defined by

$$
W_{0}(z)=-\log \frac{\sigma_{1}(z) \sigma_{2}(z)}{\sigma_{1}^{*}(z) \sigma_{2}^{*}(z)}
$$

where $\sigma_{1}, \sigma_{2}$ and $\sigma_{1}^{*}, \sigma_{2}^{*}$ are functions of $z$ defined by the following doubly infinite products:

$$
\left.\begin{array}{l}
\sigma_{1}(z)=z \prod_{m, n=-\infty}^{\infty}\left(1-\frac{z}{P_{m n}}\right) \exp \left(\frac{z}{P_{m n}}+\frac{z^{2}}{2 P_{m n}^{2}}\right), \\
\sigma_{2}(z)=\prod_{m, n=-\infty}^{\infty}\left(1-\frac{z}{Q_{m n}}\right) \exp \left(\frac{z}{Q_{m n}}+\frac{z^{2}}{2 Q_{m n}^{2}}\right), \\
\sigma_{1}^{*}(z)=\prod_{m, n=-\infty}^{\infty}\left(1-\frac{z}{P_{m n}^{*}}\right) \exp \left(\frac{z}{P_{m n}^{*}}+\frac{z^{2}}{2 P_{m n}^{* 2}}\right), \\
\sigma_{2}^{*}(z)=\prod_{m, n=-\infty}^{\infty}\left(1-\frac{z}{Q_{m n}^{*}}\right) \exp \left(\frac{z}{Q_{m n}^{*}}+\frac{z^{2}}{2 Q_{m n}^{* 2}}\right),
\end{array}\right\}
$$

in each of which the double multiplication is extended to all positive and negative integers of $m$ and $n$ including zero, except that in the first the pair of simultaneous zeros is omitted. This omission is distinguished by adding to the product sign an accent as indicated. It may be noted that the function $\sigma_{1}$ is Weierstrass' Sigma function while the remaining three functions ${ }^{6}$ are closely allied to $\sigma_{1}$. Thus, we have

${ }^{6}$ Cf. E. T. Copson, Functions of a complex variable, Oxford University Press, 1935, p. 378. 


$$
\begin{aligned}
W_{0}(z)=-\log z & -\sum_{m, n=-\infty}^{\infty}\left\{\log \left(1-\frac{z}{P_{m n}}\right)+\frac{z}{P_{m n}}+\frac{z^{2}}{2 P_{m n}^{2}}\right\} \\
& -\sum_{m, n=-\infty}^{\infty}\left\{\log \left(1-\frac{z}{Q_{m n}}\right)+\frac{z}{Q_{m n}}+\frac{z^{2}}{2 Q_{m n}^{2}}\right\} \\
& +\sum_{m, n=-\infty}^{\infty}\left\{\log \left(1-\frac{z}{P_{m n}^{*}}\right)+\frac{z}{P_{m n}^{*}}+\frac{z^{2}}{2 P_{m n}^{*^{2}}}\right\} \\
& +\sum_{m, n=-\infty}^{\infty}\left\{\log \left(1-\frac{z}{Q_{m n}^{*}}\right)+\frac{z}{Q_{m n}^{*}}+\frac{z^{2}}{2 Q_{m n}^{* 2}}\right\},
\end{aligned}
$$

where the accent on the summation sign indicates likewise the omission of the pair of simultaneous zeros of $m$ and $n$ from the double summation. By expanding the logarithmic terms in the neighborhood of the origin, this leads to

$$
W_{0}(z)=-\log z-\sum_{k=3}^{\infty} \frac{1}{k} \Omega_{k} z^{k},
$$

where, for $k W 3$,

$$
\Omega_{k}=-\sum_{m, n=-\infty}^{\infty} \frac{1}{P_{m n}^{k}}+\sum_{m, n=-\infty}^{\infty}\left(\frac{1}{P_{m n}^{* k}}+\frac{1}{Q_{m n}^{* k}}-\frac{1}{Q_{m n}^{k}}\right) .
$$

It is readily shown by symmetry that the coefficient $\Omega_{k}$ vanishes identically when $k$ is odd, and is real when $k$ is even.

A class of analytic functions with poles of integral orders at the foregoing sets of image points will be defined by

$$
W_{s}(z)=\frac{(-1)^{s}}{(s-1) !} \frac{d^{s}}{d z^{s}} W_{0}(z),
$$

where $s$ is the order of poles of the function. When $s \geq 2$, the class of functions are doubly periodic or elliptic functions.

The form of the functions thus derived is different according as $s$ is odd or even. The results are as follows. The initial function $W_{0}$ is also rewritten for the sake of uniformity.

and

$$
\begin{aligned}
& W_{0}(z)=-\log z-\sum_{n=2}^{\infty}{ }^{2 n} \alpha_{0} z^{2 n}, \\
& W_{2}(z)=\frac{1}{z^{2}}-\sum_{n=1}^{\infty}{ }^{2 n} \alpha_{2} z^{2 n},
\end{aligned}
$$

$$
\left.\begin{array}{c}
W_{2 s}(z)=\frac{1}{z^{2 s}}-\sum_{n=0}^{\infty}{ }^{2 n} \alpha_{2 s} z^{2 n}, \quad(s \geq 2) \\
W_{2 s+1}(z)=\frac{1}{z^{2 s+1}}+\sum_{n=0}^{\infty}{ }^{2 n+1} \alpha_{2 s+1} z^{2 n+1}, \quad(s \geq 0)
\end{array}\right\}
$$


where

and in particular

$$
\left.\begin{array}{l}
{ }^{n} \alpha_{s}=\left(\begin{array}{c}
n+s-1 \\
n
\end{array}\right) \Omega_{n+s} \\
{ }^{n} \alpha_{0}=\frac{1}{n} \Omega_{n}, \quad{ }^{1} \alpha_{1}=0 .
\end{array}\right\}
$$

Note that here the coefficient ${ }^{0} \alpha_{2}$ is not defined. It will be reserved for later usage.

Now split these functions into real and imaginary parts as follows: For $s \geq 0$,

$$
W_{\imath}(z)=S_{s}(x, y)-i T_{s}(x, y) .
$$

Also, define a pair of polar coordinates $(r, \theta)$ by

$$
z=x+i y=r e^{i \theta} \text {. }
$$

We then have, since ${ }^{n} \alpha_{s}$ is real,

$$
\left.\begin{array}{rl}
S_{0} & =-\log r-\sum_{n=2}^{\infty}{ }^{2 n} \alpha_{0} r^{2 n} \cos 2 n \theta, \\
T_{0} & =\theta+\sum_{n=2}^{\infty}{ }^{2 n} \alpha_{0} r^{2 n} \sin 2 n \theta, \\
S_{2} & =\frac{\cos 2 \theta}{r^{2}}-\sum_{n=1}^{\infty}{ }^{2 n} \alpha_{2} r^{2 n} \cos 2 n \theta, \\
S_{2 s} & =\frac{\cos 2 s \theta}{r^{2 s}}-\sum_{n=0}^{\infty}{ }^{2 n} \alpha_{2 s} r^{2 n} \cos 2 n \theta, \quad(s \geq 2) \\
T_{2 s} & =\frac{\sin 2 s \theta}{r^{2 s}}+\sum_{n=1}^{\infty}{ }^{2 n} \alpha_{2 s} r^{2 n} \sin 2 n \theta, \quad(s \geq 1) \\
S_{2 s+1} & =\frac{\cos (2 s+1) \theta}{r^{2 s+1}}+\sum_{n=0}^{\infty}{ }^{2 n+1} \alpha_{2 s+1} r^{2 n+1} \cos (2 n+1) \theta, \quad(s \geq 0) \\
T_{2 s+1} & =\frac{\sin (2 s+1) \theta}{r^{2 s+1}}-\sum_{n=0}^{\infty}{ }^{2 n+1} \alpha_{2 s+1} r^{2 n+1} \sin (2 n+1) \theta, \quad(s \geq 0)
\end{array}\right\}
$$

The preceding expressions give the expansions of the harmonic functions $S_{s}$ and $T$. in the neighborhood of the origin. Note that $S_{28}$ is even in both $x$ and $y, T_{2 s}$ is odd in both $x$ and $y, S_{2 s+1}$ is odd in $x$ but even in $y$, and $T_{2 s+1}$ is even in $x$ but odd in $y$.

It can be shown from Cauchy-Riemann differential equations that the following relations exist.

$$
\left.\begin{array}{ll}
\frac{\partial S_{0}}{\partial x}=-\frac{\partial T_{0}}{\partial y}=-S_{1}, & \frac{\partial T_{0}}{\partial x}=\frac{\partial S_{0}}{\partial y}=-T_{1}, \\
\frac{\partial S_{s}}{\partial x}=-\frac{\partial T_{s}}{\partial y}=-s S_{s+1}, & \frac{\partial T_{s}}{\partial x}=\frac{\partial S_{s}}{\partial y}=-s T_{s+1} \cdot
\end{array}\right\}
$$


Furthermore, it can be shown that for $s \geq 1$ :

(1) when $x=0, \quad \pm 2 a, \quad \pm 4 a, \cdots$,

$$
T_{2 s}=0, \quad S_{2 s+1}=0
$$

(2) when $x= \pm a, \quad \pm 3 a, \quad \pm 5 a, \cdots$,

$$
S_{2}=\text { const., } \quad S_{2 s}=0, \quad T_{2 s+1}=0 ;
$$

(3) when $y=0, \quad \pm 2 b, \quad \pm 4 b, \cdots$,

$$
T_{2 s}=0, \quad T_{2 s+1}=0 ;
$$

(4) when $y= \pm b, \quad \pm 3 b, \quad \pm 5 b, \cdots$,

$$
S_{2}=\text { const., } \quad S_{2 s}=0, \quad S_{2 s+1}=0 .
$$

The two real constants for $S_{2}$ are in fact identical and will be denoted by ${ }^{0} \alpha_{2}$. It is found that

$$
{ }^{0} \alpha_{2}=2 \wp(2 a+2 b i)
$$

where $\varphi(z)$ is Weisrstrass' elliptic function of double periods $4 a$ and $4 b i$. It is seen that the functions $S_{2 s}$ all vanish on the external boundary of the given rectangle, i.e., at $x= \pm a$ and $y= \pm b$, except $S_{2}$ which becomes a constant. Therefore, the system of functions is useful in constructing the function $\psi_{1}$.

The solution. For a solid rectangular prism of cross section $2 a \times 2 b$, the function $\psi_{0}$ is known $\operatorname{as}^{7}$

$$
\begin{aligned}
\psi_{0}=b^{2} & +\frac{1}{2}\left(x^{2}-y^{2}\right) \\
& -4 b^{2}\left(\frac{2}{\pi}\right)^{3} \sum_{n=0}^{\infty} \frac{(-1)^{n}}{(2 n+1)^{3}} \frac{\cosh (2 n+1) \pi x / 2 b}{\cosh (2 n+1) \pi a / 2 b} \cos \frac{(2 n+1) \pi y}{2 b},
\end{aligned}
$$

the origin being at the centre of the rectangular section. With the aid of the expansion

$$
\cosh p x \cos p y=\sum_{m=0}^{\infty} \frac{1}{(2 m) !}(p r)^{2 m} \cos 2 m \theta
$$

the above function may be expressed in polar coordinates as follows:

where, for $n \geq 0$,

$$
\psi_{0}=f_{0}+\sum_{n=1}^{\infty} f_{2 n} r^{2 n} \cos 2 n \theta
$$

$$
f_{2 n}=b^{2} \delta_{0, n}+\frac{1}{2} \delta_{1, n}-\frac{4}{b(2 n) !} \sum_{m=0}^{\infty}(-1)^{m}\left\{\frac{(2 m+1) \pi}{2 b}\right\}^{2 n-3} \operatorname{sech} \frac{(2 m+1) \pi a}{2 b}
$$

in which $\delta m n=1$ or 0 , according as $m=n$, or $m \neq n$.

Now, we construct

${ }^{7}$ A. E. H. Love, Mathematical theory of elasticity, 4th ed., Dover Publications, 1944, 317-318. Note that the constant is modified so that $\psi_{0}=\frac{1}{2}\left(x^{2}+y^{2}\right)$ on the boundary. 


$$
\psi_{1}=\sum_{s=1}^{\infty} A_{2 s} S_{2 s}(x, y)-{ }^{0} \alpha_{2} A_{2},
$$

where $A_{2 s}$ are arbitrary constants to be determined. The initial function $S_{0}$ is rejected on the ground that its conjugate function is not single-valued. The function $\psi_{1}$ thus constructed evidently meets all the requirements as outlined previously.

To adjust the remaining condition on the internal boundary or the central hole, we have in terms of polar coordinates,

$$
\begin{aligned}
\Psi=- & \frac{1}{2}\left(x^{2}+y^{2}\right)+\psi_{0}+\psi_{1} \\
=- & \frac{1}{2} r^{2}+f_{0}-\sum_{s=1}^{\infty}{ }^{0} \alpha_{2 s} A_{2 s} \\
& \quad+\sum_{n=1}^{\infty}\left(f_{2 n} r^{2 n}+A_{2 n} r^{-2 n}-r^{2 n} \sum_{s=1}^{\infty}{ }^{2 n} \alpha_{2 s} A_{2 s}\right) \cos 2 n \theta .
\end{aligned}
$$

Hence the boundary condition on the rim of the circular hole, where $r=\lambda$ say, is satisfied provided that for $n \geq 1$,

$$
f_{2 n} \lambda^{2 n}+A_{2 n} \lambda^{-2 n}-\lambda^{2 n} \sum_{s=1}^{\infty}{ }^{2 n} \alpha_{2 s} A_{2 s}=0
$$

or

$$
A_{2 n}=-f_{2 n} \lambda^{4 n}+\lambda^{4 n} \sum_{s=1}^{\infty}{ }^{2 n} \alpha_{2 s} A_{2 s} .
$$

The value of $\Psi$ on the rim of hole then becomes a constant, say $\Psi_{0}$, as follows:

$$
\Psi_{0}=-\frac{1}{2} \lambda^{2}+f_{0}-\sum_{s=1}^{\infty}{ }^{0} \alpha_{2 s} A_{2 s} .
$$

The system of linear equations in (25) may be solved by successive approximations as follows. Write

where

$$
A_{2 n}=\sum_{p=0}^{\infty} A_{2 n}^{(p)}
$$

and, by iteration,

$$
A_{2 n}^{(0)}=-f_{2 n} \lambda^{4 n}
$$

$$
A_{2 n}^{(p)}=\lambda^{4 n} \sum_{s=1}^{\infty}{ }^{2 n} \alpha_{2 s} A_{2 s}^{(p-1)} .
$$

Naturally, the validity of the solution depends upon the convergence of the series (27). From physical considerations alone, it seems likely that there will be convergence as long as the rim of hole does not touch the external boundary, i.e., when

$$
\lambda<\min (a, b) .
$$

Torsional modulus. The torsional modulus ${ }^{8}$ of the prism is given by

$$
H=2 \iint \Psi d x d y+2 \pi \lambda^{2} \Psi_{0},
$$

${ }^{8}$ R. V. Southwell, Theory of elasticity, 2nd ed., Oxford University Press, 1941, p. 323. 
where the double integration is extended over the entire rectangular section, excluding the hole.

There is no difficulty in evaluating the following integrals:

$$
\begin{aligned}
& \iint \psi_{0} d x d y=4 \int_{0}^{b} \int_{0}^{a} \psi_{0} d x d y-2 \int_{0}^{\lambda} \int_{0}^{\pi} \psi_{0} r d \theta d r \\
&=\frac{2}{3} a b\left(a^{2}+5 b^{2}\right)-\pi \lambda^{2} f_{0}-\frac{1}{2}\left(\frac{4}{\pi}\right)^{5} b^{4} \sum_{n=0}^{\infty} \frac{1}{(2 n+1)^{5}} \tanh \frac{(2 n+1) \pi a}{2 b}, \\
& \iint\left(x^{2}+y^{2}\right) d x d y=4 \int_{0}^{b} \int_{0}^{a}\left(x^{2}+y^{2}\right) d x d y-2 \int_{0}^{\lambda} \int_{0}^{\pi} r^{3} d \theta d r \\
&=\frac{4}{3} a b\left(a^{2}+b^{2}\right)-\frac{1}{2} \pi \lambda^{4} .
\end{aligned}
$$

But in evaluating the integral $\iint \psi_{1} d x d y$ the foregoing method fails owing to the fact that $\psi_{1}$ possesses a singularity at the origin. However, it may be evaluated by means

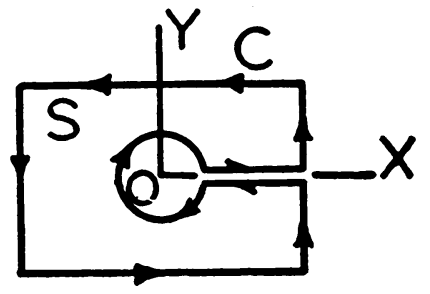

Fig. 7.

of contour integration based upon a corollary from Green's theorem. Suppose that $F$ is a function of $z$ regular in a domain $S$ which is enclosed by a contour $C$. Then we find

$$
\iint_{S} \frac{d F}{d z} d S=\frac{1}{2} i \int_{C} F d \bar{z}
$$

where $\bar{z}$ is the conjugate of $z$; the contour being taken in a counter-clockwise direction: Now, put $F=W_{s}(z)$ where $s \geq 1$. Then

$$
\iint_{S} W_{s+1}(z) d S=-\frac{i}{2 s} \int_{C} W_{s}(z) d \bar{z} .
$$

Thus by referring to the contour $C$ in Fig. 7, for $s \geq 2$,

$$
\begin{aligned}
\iint S_{2 s} d x d y & =R \cdot P \cdot \iint_{S} W_{2 s}(z) d S=-\frac{1}{2(2 s-1)} R \cdot P \cdot \int_{C} i W_{2 s-1}(z) d \bar{z} \\
& =-\frac{4}{(2 s-1)(2 s-2)} T_{2 s-2}(a, b)+\pi \Omega_{2 s} \lambda^{2} .
\end{aligned}
$$


In particular, for $s=1$,

$$
\iint S_{2} d x d y=-\frac{1}{2} R \cdot P \cdot \int_{C} i W_{1}(z) d \bar{z}=-4 T_{0}(a, b) .
$$

Consequently, we have

$$
\begin{aligned}
\iint \psi_{1} d x d y=- & A_{2}\left\{4 T_{0}(a, b)+\left(4 a b-\pi \lambda^{2}\right)^{0} \alpha_{2}\right\} \\
& \quad-\sum_{s=2}^{\infty} A_{2 s}\left\{\frac{4}{(2 s-1)(2 s-2)} T_{2 s-2}(a, b)-\pi \Omega_{2 s} \lambda^{2}\right\} .
\end{aligned}
$$

Hence the torsional modulus is equal to

$$
\begin{aligned}
H=\frac{16}{3} a b^{3}-\frac{1}{2} \pi \lambda^{4}-\left(\frac{4}{\pi}\right)^{5} b^{4} \sum_{n=0}^{\infty} \frac{1}{(2 n+1)^{5}} \tanh \frac{(2 n+1) \pi a}{2 b} \\
-2 A_{2}\left\{4 T_{0}(a, b)+\left(4 a b-\pi \lambda^{2}\right)^{0} \alpha_{2}\right\}-8 \sum_{s=1}^{\infty} \frac{1}{2 s(2 s+1)} A_{2 s+2} T_{2 s}(a, b) .
\end{aligned}
$$

The resulting twisting couple is given by

$$
T=\mu \tau H
$$

where $\mu$ is the modulus of rigidity of the material and $\tau$ is the angle of twist per unit length of the prism.

Stress components. The non-vanishing stress components of the prism are two shear stress components given in terms of rectangular coordinates by

$$
Z_{x}=\mu \tau \frac{\partial \Psi}{\partial y}, \quad Z_{\nu}=-\mu \tau \frac{\partial \Psi}{\partial x}
$$

or in terms of polar coordinates by

$$
Z_{r}=\frac{\mu \tau}{r} \frac{\partial \Psi}{\partial \theta}, \quad Z_{\theta}=-\mu \tau \frac{\partial \Psi}{\partial r}
$$

It is now a straightforward matter to calculate the stress at any point in the prism. In particular the shear stresses on the external and internal boundaries are as follows:

$$
\begin{aligned}
& \left.\left[Z_{x}\right]_{y=b}=-2 \mu \tau\left\{b-\frac{8 b}{\pi^{2}} \sum_{n=0}^{\infty} \frac{\cosh (2 n+1) \pi x / 2 b}{(2 n+1)^{2} \cosh (2 n+1) \pi a / 2 b}+\sum_{s=1}^{\infty} s A_{2 s} T_{2 s+1}(x, b)\right\}\right) \\
& {\left[Z_{y}\right]_{x=-}=2 \mu \tau\left\{\frac{8 b}{\pi^{2}} \sum_{n=0}^{\infty} \frac{(-1)^{n}}{(2 n+1)^{2}} \tanh \frac{(2 n+1) \pi a}{2 b} \cos \frac{(2 n+1) \pi y}{2 b}\right.} \\
& \left.\quad+\sum_{s=1}^{\infty} s A_{2 s} S_{2 s+1}(a, y)\right\} \\
& {\left[Z_{\theta}\right]_{r=\lambda}=\mu \tau\left(\lambda+4 \sum_{n=1}^{\infty} \frac{n A n}{\lambda^{2 n+1}} \cos 2 n \theta\right)}
\end{aligned}
$$


The maximum shear stress occurs on the boundary at those points which are nearest to the next boundary. Due to the presence of the holes, the greatest shear stress does not necessarily occur on the external boundary.

Evaluation of coefficients. Numerical results will be given for the case of a square prism of cross section $2 a \times 2 a$ (i.e., $a=b$ ) having a central hole of two different radii $\lambda=a / 2$ and $a / 3$ respectively.

When $a=b$, it can be further shown that for $k \geq 1$,

where

$$
\begin{aligned}
\Omega_{4 k+2} & =0, \\
\Omega_{4 k} & =\frac{1}{(2 a)^{4 k}}\left(1-\frac{2(-1)^{k}}{2^{2 k}}\right) \sigma_{4 k},
\end{aligned}
$$

$$
\sigma_{4 k}=\sum_{m, n=-\infty}^{\infty} \frac{1}{(m+n i)^{4 k}} .
$$

There exists an algebraic relation between the coefficients ${ }^{9} \sigma_{4 k}$. For $k \geq 2$,

$$
\frac{1}{3}(2 k-3)(4 k+1) C_{4 k}=C_{4} C_{4 k-4}+C_{8} C_{4 k-8}+C_{12} C_{4 k-12}+\cdots+C_{4 k-4} C_{4}
$$

where, for $k \geq 1$,

$$
C_{4 k}=(4 k-1) \sigma_{4 k}
$$

The values of $\sigma_{4 k}$ have been computed by the present writer some time ago. ${ }^{10}$ They Table I. Cogfficients for a Square Prism

\begin{tabular}{r|c|c|c|c|c}
\hline \hline$n$ & $\sigma_{4 n}$ & $\Omega_{4 n}(2 a)^{4 n}$ & $f_{2 n} a^{2 n-2}$ & $\frac{A_{2 n}}{a^{2 n+2}}$ for $\lambda=\frac{a}{2}$ & $\frac{A_{2 n}}{a^{2 n+2}}$ for $\lambda=\frac{a}{3}$ \\
\hline 0 & - & - & $8.93704 \times 10^{-2}$ & - & - \\
1 & 3.151212 & 4.726818 & 0 & 0 & 0 \\
2 & 4.255773 & 3.723801 & $-9.11851 \times 10^{-2}$ & $3.56902 \times 10^{-4}$ & $1.38992 \times 10^{-6}$ \\
3 & 3.938849 & 4.061938 & 0 & 0 & 0 \\
4 & 4.015695 & 3.984322 & $1.92418 \times 10^{-3}$ & $-2.84698 \times 10^{-8}$ & $-4.46471 \times 10^{-11}$ \\
5 & 3.996097 & 4.003902 & 0 & 0 & 0 \\
6 & 4.000977 & 3.999023 & $-1.20581 \times 10^{-4}$ & $7.77531 \times 10^{-12}$ & $4.28303 \times 10^{-16}$ \\
7 & 3.999756 & 4.000244 & 0 & 0 & 0 \\
8 & 4.000061 & 3.999939 & $1.1841 \times 10^{-5}$ & $-2.4495 \times 10^{-15}$ & $-6.3624 \times 10^{-21}$ \\
9 & 3.999985 & 4.000015 & 0 & 0 & 0 \\
10 & 4.000004 & 3.999996 & $-1.4543 \times 10^{-6}$ & $1.4594 \times 10^{-18}$ & $1.2010 \times 10^{-25}$ \\
11 & 3.999999 & 4.000001 & 0 & 0 & 0 \\
12 & 4.000000 & 4.000000 & $2.0472 \times 10^{-7}$ & $-6.7229 \times 10^{-22}$ & $-2.5589 \times 10^{-80}$ \\
\hline
\end{tabular}

are reproduced in Table I where values of $\Omega_{4 k}$ and $f_{4 n}$ are also tabulated. It is noted that by symmetry, for $n \geq 0$,

$$
f_{4 n+2}=0, \quad A_{4 n+2}=0 .
$$

${ }^{9}$ Cf. E. T. Copson, loc. cit., p. 360.

${ }^{10}$ Doctoral thesis by C. B. Ling presented to London University, England (1937). 
Values of ${ }^{4 n} \alpha_{48}$ are shown in Table II. With these values, the coefficients $A_{4 n}$ can now be computed from (27) by successive approximations. The results for two different radii $\lambda=a / 2$ and $a / 3$ are shown together in Table $\mathrm{I}$.

TABLe II. ${ }^{4 n} \alpha_{48} a^{4 n+48}$ For A SQUARe Prism

\begin{tabular}{r|c|c|c|c|c|c}
\hline \multicolumn{1}{r|}{$4 s$} & $4 n=4$ & $4 n=8$ & $4 n=12$ & $4 n=16$ & $4 n=20$ & $4 n=24$ \\
\hline 4 & $5.09114 \times 10^{-1}$ & $1.63628 \times 10^{-1}$ & $2.76622 \times 10^{-2}$ & $3.70005 \times 10^{-3}$ & $4.22136 \times 10^{-4}$ & $4.35886 \times 10^{-5}$ \\
8 & $3.27256 \times 10^{-1}$ & $3.91222 \times 10^{-1}$ & $1.92402 \times 10^{-1}$ & $5.84357 \times 10^{-2}$ & $1.32335 \times 10^{-2}$ & $2.44895 \times 10^{-3}$ \\
12 & $8.29864 \times 10^{-2}$ & $2.88604 \times 10^{-1}$ & $3.22282 \times 10^{-1}$ & $1.94292 \times 10^{-1}$ & $7.88560 \times 10^{-2}$ & $2.42858 \times 10^{-2}$ \\
16 & $1.48002 \times 10^{-2}$ & $1.16871 \times 10^{-1}$ & $2.59056 \times 10^{-1}$ & $2.79896 \times 10^{-1}$ & $1.89056 \times 10^{-1}$ & $9.14617 \times 10^{-2}$ \\
20 & $2.11068 \times 10^{-3}$ & $3.30837 \times 10^{-2}$ & $1.31427 \times 10^{-1}$ & $2.36320 \times 10^{-1}$ & $2.50741 \times 10^{-1}$ & $1.82006 \times 10^{-1}$ \\
24 & $2.61531 \times 10^{-4}$ & $7.34684 \times 10^{-3}$ & $4.85717 \times 10^{-2}$ & $1.37193 \times 10^{-1}$ & $2.18408 \times 10^{-1}$ & $2.29133 \times 10^{-1}$ \\
\hline
\end{tabular}

To proceed further in computing the torsional modulus and shear stresses on the boundaries, values of ${ }^{0} \alpha_{2}, T_{4 s+2}(a, a)$ and $T_{4 s+1}(x, a)$ are required.

It is readily shown that when $a=b$,

$$
{ }^{0} \alpha_{2}=0 \text {. }
$$

Now, define similarly a class of analytic functions $W_{s}^{*}(z)$ whose initial function is

$$
W_{0}^{*}(z)=-\log \left\{\sigma_{1}(z) \sigma_{2}(z)\right\},
$$

where $\sigma_{1}$ and $\sigma_{2}$ are defined before in (6). The expansion of $W_{s}^{*}(z)$ in the neighborhood of the origin, for $s \geq 1$, is

$$
W_{s}^{*}(z)=\frac{(-1)^{s}}{(s-1) !} \frac{d^{s}}{d z^{s}} W_{0}^{*}(z)=\frac{1}{z^{s}}+(-1)^{s} \sum_{n=0}^{\infty}{ }^{n} \alpha_{s}^{*} z^{n},
$$

where, for $a=b$,

$$
{ }^{n} \alpha_{s}^{*}=(-1)^{(n+s) / 4}\left(\begin{array}{c}
n+s-1 \\
n
\end{array}\right) \frac{\sigma_{n+s}}{(2 / 2 a)^{n+s}} .
$$

$\sigma_{n+s}$ is defined in (44), which vanishes unless the suffix $(n+s)$ is an integral multiple of 4 . An algebraic relation between the functions is as follows: For $s \geq 5$,

$$
\frac{1}{6}(s-3)(s-2) B_{s}=B_{2} B_{s-2}+B_{3} B_{s-3}+B_{4} B_{s-4}+\cdots+B_{s-2} B_{2}
$$

where, for $s \geq 2$,

$$
B_{s}=(s-1) W_{s}^{*}(z)=(s-1)\left\{S_{s}^{*}(x, y)-i T_{s}^{*}(x, y)\right\}
$$

When $a=b$, it can be further shown that for $s \geq 1$,

$$
\begin{aligned}
W_{2 s+1}^{*}(a+a i) & =0, \\
W_{4 s}^{*}(a+a i) & =S_{4 s}^{*}(a, a), \\
W_{4 s-2}^{*}(a+a i) & =-i T_{4 s-2}^{*}(a, a)=-\frac{1}{2} i T_{4 s-2}(a, a) .
\end{aligned}
$$


The two initial functions may be found from

$$
\begin{aligned}
& W_{2}^{*}(a+a i)=-\frac{i\left(1 \frac{\left.5 \sigma_{4}\right)^{1 / 2}}{8 a^{2}},\right.}{W_{4}^{*}(a+a i)=-\frac{5 \sigma_{4}}{32 a^{4}} .}
\end{aligned}
$$

With the aid of (57) for successive functions, values of $T_{4 s+2}(a, a)$ can then be found without difficulty.

To evaluate $T_{4 s+1}(x, a)$, it is noted that for $a=b$,

$$
T_{4 s+1}(x, a)=2 T_{4 s+1}^{*}(x, a)=R . P .\left\{2 i W_{4 s+1}^{*}(x+i a)\right\}
$$

In general, the three initial functions of $W_{s}^{*}(z)$ may be found from

$$
\begin{aligned}
& W_{2}^{*}(z)=\left\{\frac{W_{3}^{*}(x)-i W_{3}^{*}(y)}{W_{2}^{*}(x)+W_{2}^{*}(y)}\right\}^{2}-W_{2}^{*}(x)+W_{2}^{*}(y), \\
& W_{3}^{*}(z)=\left[\left\{W_{2}^{*}(z)\right\}^{3}+\frac{15 \sigma_{4}}{64 a^{4}} W_{2}^{*}(z)\right]^{1 / 2}, \\
& W_{4}^{*}(z)=\left\{W_{2}^{*}(z)\right\}^{2}+\frac{5 \sigma_{4}}{64 a^{4}} .
\end{aligned}
$$

\begin{tabular}{|c|c|c|c|c|c|c|c|}
\hline \multirow{2}{*}{$s$} & \multirow{2}{*}{$T_{48-2}(a, a)$} & \multicolumn{6}{|c|}{$T_{4 s+1}(x, a)$} \\
\hline & & $x=0$ & $x=0.2 a$ & $x=0.4 a$ & $x=0.6 a$ & $x=0.8 a$ & $x=a$ \\
\hline 1 & 1.71880 & 1.93656 & 0.46903 & -0.25063 & -0.43000 & -0.25138 & 0 \\
\hline 2 & -0.50778 & 2.00239 & -0.16957 & -0.48920 & 0.04270 & 0.10612 & 0 \\
\hline 3 & 0.12501 & & & & & & \\
\hline
\end{tabular}

With also the aid of (55) and (57), values of $T_{4 s+1}(x, a)$ can then be computed for any particular value of $x$.

The results are tabulated in Tables III, IV and V.

TABLe III. $T_{48-2}(a, a)$ and $T_{48+1}(x, a)$ For A Square Prism

Table IV. Cross Sectional Area $A$ and Torsional Modulus $H$ of a Square Prism

\begin{tabular}{c|c|c|c|c}
\hline \hline$\lambda / a$ & $A / a^{2}$ & $H / a^{4}$ & \% reduction of $A$ & \% reduction of $H$ \\
\hline$\frac{1}{2}$ & 3.2146 & 2.1502 & 19.63 & 4.40 \\
$\frac{1}{2}$ & 3.6509 & 2.2298 & 8.73 & 0.86 \\
Solid & 4.0000 & 2.2492 & 0 & 0 \\
\hline
\end{tabular}


Table V. Shear Stresses on Boundaries of a Square Prism

\begin{tabular}{|c|c|c|c|c|c|c|}
\hline \multicolumn{4}{|c|}{$Z_{x} / \mu \tau a$ on $y=a$} & \multicolumn{3}{|c|}{$Z_{\theta} / \mu \tau a$ on $r=\lambda$} \\
\hline$x / a$ & $\lambda=\frac{1}{2} a$ & $\lambda=\frac{1}{3} a$ & Solid & $\theta^{\circ}$ & $\lambda=\frac{1}{2} a$ & $\lambda=\frac{1}{3} a$ \\
\hline 0 & -1.353 & -1.351 & -1.351 & 0 & 0.524 & 0.360 \\
\hline 0.2 & -1.316 & -1.315 & -1.315 & 5 & 0.523 & 0.359 \\
\hline 0.4 & -1.210 & -1.211 & -1.211 & 10 & 0.518 & 0.354 \\
\hline 0.6 & -1.014 & -1.014 & -1.014 & 20 & 0.504 & 0.338 \\
\hline 0.8 & -0.683 & -0.684 & -0.684 & 30 & 0.488 & 0.320 \\
\hline 1 & 0 & 0 & 0 & 45 & 0.476 & 0.306 \\
\hline
\end{tabular}

The writer wishes to thank Mr. T. C. Lee of the Aeronautical Research Laboratory, Taiwan, for his assistance in preparing the manuscript. 